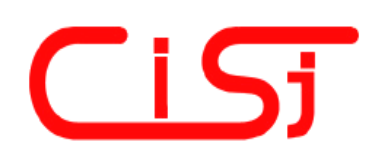

\title{
PROBABILISTIC PROPERTIES AND EVALUATION OF DESCRIPTIONS OF INITIAL SIGNAL OF NONLINEAR SYSTEM AT ACTION OF PERIODIC WHITE NOISE
}

\author{
Sergiy Lupenko, Mykola Prijmak
}

\author{
Ternopil Technical University, \\ Department of Computer Sciences, \\ 54 Ruska street, Ternopil 46001, Ukraine
}

Keywords: design, nonlinear system, stochastic periodic casual process, statistical estimations.

\section{INTRODUCTION}

At development and creation of the informative dynamic systems often there are the tasks of their imitation design and analysis of signals, that take place in such systems. Possibility of decision of similar tasks relies on adequacy and efficiency of the use of mathematical models of the explored dynamic systems substantially. The tasks of imitation design and analysis of nonlinear transformations stochastic of batch casual processes turn out especially difficult. Complication is conditioned not only nonlinear of the dynamic system but also unstationary of entrance casual process.

Similar tasks of analysis were examined for the cases, when the system is nonlinear, and an entrance casual process is stationary $[1,2]$, and when the dynamic system is linear, and its entrance process is stochastic periodic [3]. In addition in work [3] it is led to, that when the entrance process of the linear time-invariant system is a periodic white noise in the wide understanding, on the return of such system there will be the periodically correlated linear casual process, that is periodic within the framework of the first two moment functions. In $[4,5]$ this question it is considered within the framework of all moment functions and multidimensional characteristic function of linear casual process. In work [6] it is led to $T$-periodic mathematical hope and correlation function of the stochastic time-invariant Volterra second order of nonlinear functional to the polynomial for the case, when its input process is a $T$-periodic white noise.

The given article extends results, that are got in work [6], by leading of assertion about $T$-periodic to initial higher moment functions of stochastic nonlinear to time-invariant functional Volterra in case of random of nonlinear at the same condition, when on the entrance of the nonlinear dynamic system operates a $T$-periodic white noise.

\section{BASIC PART}

In practice at research of the nonlinear system, it is given as a stochastic time-invariant polynomial:

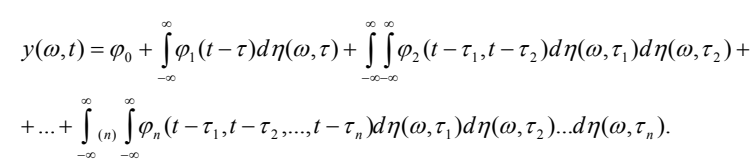

How it was marked higher, in work [6], that when in (1) a casual process $\eta(\omega, \tau)$ is a process with independent $T$-periodic increases, a mathematical hope and correlation function of review of the second order of nonlinear stochastic to the polynomial (1) also will be $T$-periodic. It was considered thus, that a linear element in a polynomial (1) was necessarily present.

It is formulated and a next theorem is proved.

Theorem 1. If for a stochastic functional to the polynomial (1) a periodic white noise with a period is its input process, initial moment functions to the polynomial (1) will be periodic with the same period $T$.

For stochastic $T$-periodic processes in the narrow understanding characteristic such property: counting of process, taken through a period $T$ form casual sequences stationary and stationary linked in the narrow understanding, out. 
If there are the moment functions of stochastic $T$-periodic casual process initial and central, they are $T$-periodic, that is a process is stochastic $T$ periodic moment functions relatively initial and central.

On the basis of theorem we will write down statistical estimations of moment functions initial and central stochastic $T$-periodic relatively higher moment functions of nonlinear functional to the polynomial (1). Lets we have realization $y(t), \quad t \in \mathbf{M}$ stochastic of $T$-periodic casual process, $M$ - length of interval of supervision $\mathbf{M}$.

Statistics are estimation of initial moment function of $k$-th order in the moment of time $t \in \mathbf{T}$

$$
\hat{m}_{k}(t)=\frac{1}{Z} \cdot \sum_{j=0}^{Z-1} y^{k}(t+j \cdot T), \quad t \in \mathbf{T}
$$

where $Z=\left[\frac{M}{T}\right]$ - amount of the registered cycles of realization $y(t), t \in \mathbf{M}$, that the supervisions after a signal to the size of period are determined as whole part from the division of all time .

Estimation of central moment function of $k$-th order

$$
\hat{d}_{k}(t)=\frac{1}{Z-1} \cdot \sum_{j=0}^{Z-1}\left(y_{\omega}(t+j \cdot T)-\hat{m}_{1}(t)\right)^{k}, \quad t \in \mathbf{T} \cdot(3)
$$

Statistical estimations (2) are resulted and (3) in particular can be used for finding of estimations of mathematical hope and dispersion of nonlinear stochastic periodic functional.

On the basis of theorem the statistical methods of treatment and analysis of casual process are developed, that is observed on the return of the nonlinear dynamic system, when on its entrance operates a $T$-periodic white noise.

\section{CONCLUSION}

It is led to, that higher moment functions initial and central of stochastic functional to the polynomial (1) are periodic with a period even to the period of increases of process with independent periodic increases. This fact grounds to apply the statistical methods of treatment and analysis stochastic of periodic signals which take place in the nonlinear systems, within the framework of higher moment functions of casual processes. Statistical estimations of moment functions of initial process of the nonlinear time-invariant system initial and central are resulted at influence on it of periodic white noise. Except for this image (1) is the structural model of the explored signals, that enables to conduct their imitation design by the tools of the computing engineering. 


\title{
ЙМОВІРНІСНІ ВЛАСТИВОСТІ ТА ОЦІНЮВАННЯ ХАРАКТЕРИСТИК ВИХІДНОГО СИГНАЛУ НЕЛІНІЙНОЇ СИСТЕМИ ПРИ ДІЇ ПЕРІОДИЧНОГО БІЛОГО ШУМУ
}

\author{
Сергій Лупенко, Микола Приймак
}

\author{
Тернопільський державний технічний університет, \\ Кафедра комп'ютерних наук, \\ вул. Руська 54, м. Тернопіль 46001, Україна
}

\begin{abstract}
Резюме: У роботі доведено теорему про періодичність вищих моментних функиій вихідного процесу часоінваріантної нелінійної системи, при умові, якщо на ї̈ вхід діє періодичний білий шум. Обгрунтовано статистичні методи оцінювання періодичних моментних функиій вихідного процесу нелінійної системи
\end{abstract}

Ключові слова: дизайн, нелінійна система, стохастичний періодичний нерегулярний процес, статистичні оцінки.

\section{1. ВСТУП}

При розробці та створенні інформаційних динамічних систем часто виникають задачі їх імітаційного моделювання та аналізу сигналів, що мають місце в таких системах. Можливість розв'язання подібних задач суттєво залежить від адекватності та ефективності використання математичних моделей досліджуваних динамічних систем. Особливо складними виявляються задачі імітаційного моделювання та аналізу нелінійних перетворень стохастично періодичних випадкових процесів. Складність обумовлена не тільки нелінійністю динамічної системи, але i нестаціонарністю вхідного випадкового процесу.

Подібні задачі аналізу розглядалися для випадків, коли система є нелінійною, а вхідний випадковий процес $є$ стаціонарним $[1,2]$, та коли динамічна система $\epsilon$ лінійною, а ii вхідний процес $є$ стохастично періодичним [3]. Крім того в роботі [3] доведено, що коли вхідний процес лінійної часоінваріантної системи $є$ періодичним білим шумом у широкому розумінні, то на виході такої системи буде спостерігатися періодично корельований лінійний випадковий процес, тобто періодичний в рамках перших двох моментних функцій. $\mathrm{B}[4,5]$ це питання розглянуто в рамках всіх моментних функцій та багатовимірної характеристичної функції лінійного випадкового процесу. В роботі [6] доведено $T$-періодичність математичного сподівання та кореляційної функції стохастичного часоінваріантного функціонального поліному Вольтерра другого порядку нелінійності для випадку, коли його породжуючий процес є $T$-періодичним білим шумом.

Дана стаття розширює результати, що отримані в роботі [6], шляхом доведення твердження про $T$-періодичність початкових вищих моментних функцій стохастичного нелінійного часоінваріантного функціоналу Вольтерра на випадок довільного скінченного порядку нелінійності при цій же умові, коли на вхід нелінійної динамічної системи діє $T$ періодичний білий шум.

\section{2. ОСНОВНА ЧАСТИНА}

Значного поширення для опису нелінійних систем набули функціональні ряди Вольтерра, Вінера [7, 8, 9] та ряди ортогональних стохастичних функціоналів [10]. Конструктивною моделлю, яка у своїй структурі відображає фізику утворення сигналу на виході нелінійної системи при дії випадкових сигналів на iii вході, $\epsilon$ ряд стохастичних функціоналів Вольтерра виду 


$$
\begin{aligned}
& y(\omega, t)=\varphi_{0}+\int_{-\infty}^{\infty} \varphi_{1}(\tau, t) d \eta(\omega, \tau)+\int_{-\infty-\infty}^{\infty} \int_{-\infty}^{\infty} \varphi_{2}\left(\tau_{1}, \tau_{2}, t\right) d \eta\left(\omega, \tau_{1}\right) d \eta\left(\omega, \tau_{2}\right)+ \\
& +\ldots+\int_{-\infty}^{\infty} \int_{(n)}^{\infty} \int_{-\infty} \varphi_{n}\left(\tau_{1}, \tau_{2}, \ldots, \tau_{n}, t\right) d \eta\left(\omega, \tau_{1}\right) d \eta\left(\omega, \tau_{2}\right) \ldots d \eta\left(\omega, \tau_{n}\right)+\ldots,
\end{aligned}
$$

де

$\left\{\varphi_{0}, \varphi_{1}(\tau, t), \varphi_{2}\left(\tau_{1}, \tau_{2}, t\right), \ldots, \varphi_{n}\left(\tau_{1}, \tau_{2}, \ldots, \tau_{n}, t\right), \ldots\right\}$

множина дійсних невипадкових інтегровних 3 квадратом функцій, що називаються ядрами Вольтерра і $\epsilon$ аналогами імпульсної реакції лінійної динамічної системи; $\eta(\omega, \tau)$ стохастично неперервний сепарабельний випадковий процес 3 незалежними приростами
[11], узагальнена похідна від якого є процесом із незалежними значеннями, тобто білим шумом.

Часто розглядають нелінійні системи, функціонування яких не змінюється в часі, тобто нелінійні часоінваріантні системи. У такому випадку ядра функціоналів $\left\{\varphi_{0}, \varphi_{1}(\tau, t), \varphi_{2}\left(\tau_{1}, \tau_{2}, t\right), \ldots, \varphi_{n}\left(\tau_{1}, \tau_{2}, \ldots, \tau_{n}, t\right), \ldots\right\}$ в (1) залежать від різниці їх аргументів, а математична модель нелінійної системи замість (1) приймає вигляд

$$
\begin{aligned}
& y(\omega, t)=\varphi_{0}+\int_{-\infty}^{\infty} \varphi_{1}(t-\tau) d \eta(\omega, \tau)+\int_{-\infty-\infty}^{\infty} \int_{2}^{\infty} \varphi_{2}\left(t-\tau_{1}, t-\tau_{2}\right) d \eta\left(\omega, \tau_{1}\right) d \eta\left(\omega, \tau_{2}\right)+ \\
& +\ldots+\int_{-\infty}^{\infty}{ }_{(n)} \int_{-\infty}^{\infty} \varphi_{n}\left(t-\tau_{1}, t-\tau_{2}, \ldots, t-\tau_{n}\right) d \eta\left(\omega, \tau_{1}\right) d \eta\left(\omega, \tau_{2}\right) \ldots d \eta\left(\omega, \tau_{n}\right)+\ldots .
\end{aligned}
$$

На практиці при дослідженні нелінійних систем, як правило, використовують лише скінченну кількість доданків ряду (2), тобто

розглядають стохастичний часоінваріантний поліном $n$-го порядку нелінійності:

$$
\begin{aligned}
& y(\omega, t)=\varphi_{0}+\int_{-\infty}^{\infty} \varphi_{1}(t-\tau) d \eta(\omega, \tau)+\int_{-\infty-\infty}^{\infty} \int_{2}^{\infty} \varphi_{2}\left(t-\tau_{1}, t-\tau_{2}\right) d \eta\left(\omega, \tau_{1}\right) d \eta\left(\omega, \tau_{2}\right)+ \\
& +\ldots+\int_{-\infty}^{\infty} \int_{(n)}^{\infty} \varphi_{-\infty}\left(t-\tau_{1}, t-\tau_{2}, \ldots, t-\tau_{n}\right) d \eta\left(\omega, \tau_{1}\right) d \eta\left(\omega, \tau_{2}\right) \ldots d \eta\left(\omega, \tau_{n}\right) .
\end{aligned}
$$

Як було зазначено вище, в роботі [6], що коли в (3) випадковий процес $\eta(\omega, \tau)$ є процесом 3 незалежними $T$-періодичними приростами, то математичне сподівання та кореляційна функція відгуку стохастичного поліному (3) другого порядку нелінійності також будуть $T$ періодичними. При цьому вважалося, що лінійний доданок у поліномі (3) є обов'язково присутнім.
Розглянемо тепер властивості початкових моментних функцій вищих $(k>2)$ порядків поліному (3). При цьому відмітимо, що згідно [12] для випадкового процесу з незалежними $T$ періодичними приростами моментні функції приростів процесу 3 незалежними $T$ періодичними приростами $T$-періодичні, тобто

$$
\mathbf{M}\left\{d \eta\left(\omega, \tau_{1}\right) \ldots d \eta\left(\omega, \tau_{n}\right)\right\}=\mathbf{M}\left\{d \eta\left(\omega, \tau_{1}+T\right) \ldots d \eta\left(\omega, \tau_{n}+T\right)\right\}
$$

Сформулюємо і доведемо наступну теорему.

Теорема 1. Якщо для стохастичного функціонального поліному (3) скінченного порядку його породжуючим процесом $\epsilon$ періодичний білий шум 3 періодом $T$, то початкові моментні функції поліному (3) будуть періодичними $з$ цим же періодом $T$.

Доведення. Для зручності та спрощення подальших викладок змінну $\quad \omega \in \boldsymbol{\Psi}$ (елементарна подія) будемо опускати, а поліном (3) перепишемо у вигляді

$$
y(t)=\sum_{i=0}^{n} N_{i}\{t\}
$$$$
\text { Де } N_{0}\{t\}=\varphi_{0} \text {, }
$$$$
N_{i}\{t\}=\int_{-\infty}^{\infty}{ }_{(i)} \int_{-\infty}^{\infty} \varphi_{i}\left(t-\tau_{1}, \ldots, t-\tau_{i}\right) d \eta\left(\omega, \tau_{1}\right) \ldots d \eta\left(\omega, \tau_{i}\right)
$$

,$i=\overline{1, n}$. 
Доведемо періодичність початкових моментна функція $k$ - го порядку моментних функцій. Для (3) його початкова

$$
\mathbf{M}\left\{y^{k}(t)\right\}=\mathbf{M}\left\{\left[\sum_{i=0}^{n} N_{i}\{t\}\right]^{k}\right\} .
$$

Застосувавши поліноміальну теорему до $k$-ї степені суми в (6), маємо

$$
\begin{aligned}
& \mathbf{M}\left\{y^{k}(t)\right\}=\mathbf{M}\left\{\sum_{k_{0}+k_{1}+\ldots+k_{n}=k} \frac{(n+1) !}{k_{0} ! k_{1} ! \cdot \ldots \cdot k_{n} !} \cdot\left(N_{0}\{t\}\right)^{k_{0}} \cdot\left(N_{1}\{t\}\right)^{k_{1}} \cdot \ldots \cdot\left(N_{n}\{t\}\right)^{k_{n}}\right\}= \\
& =\sum_{k_{0}+k_{1}+\ldots+k_{n}=k} \frac{(n+1) !}{k_{0} ! k_{1} ! \cdot \ldots \cdot k_{n} !} \cdot \mathbf{M}\left\{\left(N_{0}\{t\}\right)^{k_{0}} \cdot\left(N_{1}\{t\}\right)^{k_{1}} \cdot \ldots \cdot\left(N_{n}\{t\}\right)^{k_{n}}\right\}= \\
& =\sum_{k_{0}+k_{1}+\ldots+k_{n}=k} \frac{(n+1) !}{k_{0} ! k_{1} ! \ldots \cdot k_{n} !} \cdot \mathbf{M}\left\{\prod_{i=0}^{n}\left(N_{i}\{t\}\right)^{k_{i}}\right\}= \\
& =\sum_{k_{0}+k_{1}+\ldots+k_{n}=k} \frac{(n+1) !}{k_{0} ! k_{1} ! \ldots \cdot k_{n} !} \cdot \mathbf{M}\left\{\prod_{i=0}^{n}\left(\int_{-\infty}^{\infty}(i) \int_{-\infty}^{\infty} \varphi_{i}\left(t-\tau_{1}, \ldots, t-\tau_{i}\right) d \eta\left(\tau_{1}\right) \ldots d \eta\left(\tau_{i}\right)\right)^{k_{i}}\right\}= \\
& =\sum_{k_{0}+k_{1}+\ldots+k_{n}=k} \frac{(n+1) !}{k_{0} ! k_{1} ! \ldots \cdot k_{n} !} . \\
& \cdot \mathbf{M}\left\{\prod_{i=0}^{n} \int_{-\infty}^{\infty}\left(i \cdot k_{i}\right) \int_{-\infty}^{\infty} \prod_{j=1}^{k_{i}} \varphi_{i}\left(t-\tau_{1+j \cdot i}, \ldots, t-\tau_{i+j \cdot i}\right) d \eta\left(\tau_{1}\right) \ldots d \eta\left(\tau_{i \cdot k_{i}}\right)\right\}= \\
& =\sum_{k_{0}+k_{1}+\ldots+k_{n}=k} \frac{(n+1) !}{k_{0} ! k_{1} ! \cdot \ldots \cdot k_{n} !} . \\
& \cdot \mathbf{M}\left\{\int_{-\infty}^{\infty}\left(i \cdot k_{i} \cdot n\right) \int_{-\infty}^{\infty} \prod_{i=0}^{n} \prod_{j=1}^{k_{i}} \varphi_{i}\left(t-\tau_{1+j \cdot i}, \ldots, t-\tau_{i+j \cdot i}\right) d \eta\left(\tau_{1}\right) \ldots d \eta\left(\tau_{i \cdot k_{i} \cdot n}\right)\right\}= \\
& =\sum_{k_{0}+k_{1}+\ldots+k_{n}=k} \frac{(n+1) !}{k_{0} ! k_{1} ! \cdot \ldots \cdot k_{n} !} . \\
& \cdot \int_{-\infty}^{\infty}\left(i \cdot k_{i} \cdot n\right) \int_{-\infty}^{\infty} \prod_{i=0}^{n} \prod_{j=1}^{k_{i}} \varphi_{i}\left(t-\tau_{1+j \cdot i}, \ldots, t-\tau_{i+j \cdot i}\right) \mathbf{M}\left\{d \eta\left(\tau_{1}\right) \ldots d \eta\left(\tau_{i \cdot k_{i} \cdot n}\right)\right\} .
\end{aligned}
$$

Врахувуючи (4), останню рівність запишемо у вигляді

$$
\begin{gathered}
\mathbf{M}\left\{y^{k}(t)\right\}=\sum_{k_{0}+k_{1}+\ldots+k_{n}=k} \frac{(n+1) !}{k_{0} ! \cdot k_{1} ! \ldots \cdot k_{n} !} . \\
\cdot \int_{-\infty}^{\infty}\left(i \cdot k_{i} \cdot n\right) \int_{-\infty}^{\infty} \prod_{i=0}^{n} \prod_{j=1}^{k_{i}} \varphi_{i}\left(t-\tau_{1+j \cdot i}, \ldots, t-\tau_{i+j \cdot i}\right) \mathbf{M}\left\{d \eta\left(\tau_{1}+T\right) \ldots d \eta\left(\tau_{i \cdot k_{i} \cdot n}+T\right)\right\} .
\end{gathered}
$$


Зробивши в (7) заміни

$\tau_{j}+T=\widetilde{\tau}_{j}, \quad j=\overline{1, i \cdot k_{i} \cdot n}, \quad i=\overline{0, n}$, остаточно отримаємо

$$
\begin{gathered}
\mathbf{M}\left\{y^{k}(t)\right\}=\sum_{k_{0}+k_{1}+\ldots+k_{n}=k} \frac{(n+1) !}{k_{0} ! k_{1} ! \ldots \cdot k_{n} !} \\
\cdot \int_{-\infty}^{\infty}\left(i \cdot k_{i} \cdot n\right) \int_{-\infty}^{\infty} \prod_{i=0}^{n} \prod_{j=1}^{k_{i}} \varphi_{i}\left(t+T-\widetilde{\tau}_{1+j \cdot i}, \ldots, t+T-\widetilde{\tau}_{i+j \cdot i}\right) \mathbf{M}\left\{d \eta\left(\widetilde{\tau}_{1}\right) \ldots d \eta\left(\widetilde{\tau}_{i \cdot k_{i} \cdot n}\right)\right\}= \\
=\mathbf{M}\left\{y^{k}(t+T)\right\} .
\end{gathered}
$$

Остання рівність показує періодичність початкових моментних функцій $k$-го порядку поліноміального стохастичного функціоналу (3).

Теорема доведена.

Розглянемо тепер питання періодичності центральних моментних функцій $k$-го порядку поліноміального стохастичного функціоналу (3). Для цього скористаємося співвідношеням, що пов'язує центральні моментні функції із початковими:

$$
\mathbf{M}\left\{(y(t)-\mathbf{M}\{y(t)\})^{k}\right\}=\sum_{j=0}^{k} \frac{k !}{j ! \cdot(k-j) !} \cdot(-\mathbf{M}\{y(t)\})^{j} \cdot \mathbf{M}\left\{y^{k-j}(t)\right\} .
$$

Оскільки вираз (9) є сумою добутків $T$ періодичних функцій, то і значення центральної моментної функції (9) будуть точно повторюватися через число $T$. Проте у даному випадку число $T$ не можна вважати періодом функції (9), тому що можливі випадки, коли функція (9) буде мати період, який рівний одному із чисел $\frac{T}{k}, k=2,3, \ldots$, оскільки періодом функції вважається найменше додатне число, через яке значення функції точно повторюються.

На основі теореми можлива розробка статистичних методів обробки та аналізу випадкового процесу, що спостерігається на виході нелінійної динамічної системи, коли на їі вхід діє $T$-періодичний білий шум.

Для стохастично $T$-періодичних процесів у вузькому розумінні характерна така властивість: відліки процесу, взяті через період $T$ утворюють стаціонарні та стаціонарно пов'язані у вузькому розумінні випадкові послідовності. Тобто, задана на гратці 3 кроком $T$ i будь-якою початковою фазою $\varphi \in[0, T]$ вкладена послідовність

$$
\{y(\varphi+i \cdot T), \quad i \in \mathbf{Z}\}
$$

$\epsilon$ стаціонарною, а будь-які дві такі послідовності з різними початковими фазами $\epsilon$ стаціонарно пов'язаними у вузькому розумінні. Саму послідовність (10) в [3] названо $\varphi$-серією. Стаціонарність та стаціонарна пов'язаність $\varphi$ серій означає наступне.

Нехай крок дискретизації стохастично періодичного у вузькому розумінні процесу $y(\omega, t)$ визначається як

$$
h=\frac{T}{L}, \quad L>0
$$

де $L$ - кількість відліків продискретизованого процесу $y(\omega, t)$, що припадає на період $T$, а початкова фаза рівна $\varphi_{j}=j \cdot h, j=\overline{0, L-1}$. Тоді для кожної з $\varphi_{j}$-серій

$$
\left\{y_{j}(i)=y(j h+i L h), \quad j=\overline{0, L-1}, \quad i \in \mathbf{Z}\right\}
$$

$k$-вимірна інтегральна функція розподілу не залежить від будь-якого зсуву $s$ по сукупності аргументів 


$$
\begin{gathered}
F_{j_{k}}\left(x_{1}, \ldots, x_{k} ; j h+i_{1} L h, \ldots, j h+i_{k} L h\right)= \\
F_{j_{k}}\left(x_{1}, \ldots, x_{k} ; j h+i_{1} L h+s L h, \ldots, j h+i_{k} L h+s L h\right), \quad i_{1}, \ldots, i_{k} \in \mathbf{Z}, \quad s=0,1,2,3 \ldots
\end{gathered}
$$

Взаємна $k+m$-вимірна інтегральна функція розподілу для $\varphi_{j}$ і $\varphi_{p}$-серій також не залежить від будь-якого зсуву $s$ по сукупності аргументів

$$
\begin{gathered}
F_{j p_{k+m}}\left(x_{1}, \ldots, x_{k}, y_{1}, \ldots, y_{m} ; j h+i_{1} L h, \ldots, j h+i_{k+m} L h\right)= \\
=F_{j p_{k+m}}\left(x_{1}, \ldots, x_{k}, y_{1}, \ldots, y_{m} ; j h+i_{1} L h+s L h, \ldots, j h+i_{k+m} L h+s L h\right), \quad i_{1}, \ldots, i_{k+m} \in \mathbf{Z}, \quad s=0,1,2,3, \ldots
\end{gathered}
$$

Якщо існують початкові та центральні моментні функції стохастичного $T$ періодичного випадкового процесу, то вони є $T$ періодичні, тобто сам процес $є$ стохастично $T$ періодичним відносно початкових та центральних моментних функцій скінченного порядку.

Розглянуті вище властивості $\varphi_{j}$-серій стохастично $T$-періодичних випадкових процесів дають можливість розробити статистичні методи їх обробки 3 метою оцінювання початкових та центральних моментних функцій таких процесів.

Запишемо статистичні оцінки початкових та центральних моментних функцій стохастично $T$-періодичного відносно вищих моментних функцій нелінійного функціонального поліному (3). Нехай маємо реалізацію $y(t), t \in \mathbf{M}$ стохастично $T$-періодичного випадкового процесу, $M$ - довжина інтервалу спостереження M.

Оцінкою початкової моментної функції $k$-го порядку в момент часу $t \in \mathbf{T} \epsilon$ статистика

$$
\begin{array}{r}
\hat{m}_{k}(t)=\frac{1}{Z} \cdot \sum_{j=0}^{Z-1} y^{k}(t+j \cdot T), \quad t \in \mathbf{T}, \\
\text { де } Z=\left[\frac{M}{T}\right]-\text { кількість зареєстрованих }
\end{array}
$$

циклів реалізації $y(t), t \in \mathbf{M}$, що визначаються як ціла частина від ділення всього часу $M$ спостереження за сигналом до величини періода $T$.

Оцінка центральної моментної функції $k$-го порядку

$$
\hat{d}_{k}(t)=\frac{1}{Z-1} \cdot \sum_{j=0}^{Z-1}\left(y_{\omega}(t+j \cdot T)-\hat{m}_{1}(t)\right)^{k}, \quad t \in \mathbf{T} .
$$

Наведені статистичні оцінки (15) та (16) зокрема можуть бути використані при знаходженні оцінок математичного сподівання та дисперсії нелінійного стохастично періодичного функціоналу.

\section{3. ВИСНОВКИ}

Доведено, що вищі початкові та центральні моментні функції скінченного порядку стохастичного функціонального поліному (3) $є$ періодичними із періодом, рівним періоду приростів процесу з незалежними періодичними приростами. Цей факт дає підстави застосовувати статистичні методи обробки i аналізу стохастично періодичних сигналів, які мають місце в нелінійних системах, в рамках вищих моментних функцій випадкових процесів. Наведено статистичні оцінки початкових та центральних моментних функцій вихідного процесу нелінійної часоінваріантної системи при впливі на неї періодичного білого шуму. Крім цього зображення (3) є конструктивною моделлю досліджуваних сигналів, що дає можливість проводити їх імітаційне моделювання засобами обчислювальної техніки.

\section{4. ЛІТЕРАТУРА}

1. Бойко И.Ф., Марченко Б.Г. Анализ нелинейных преобразований сигналов в системах диагностики с использованием стохастических ортогональных разложений: Препр. /АН УССР. Ин-т электродинамики; №542. - К.: 1987. - 57 c.

2. Бойко I., Марченко Б. Ортогональні стохастичні функціонали в теорії нелінійних радіотехнічних кіл. // Вісник Тернопільського державного технічного університету ім. I. Пулюя. - 1997. - Т.2, Ч.2. - С. 5 - 12.

3. Приймак Н.B. Оценка периода корреляции и ее использование при исследовании стохастически периодических радиотехнических сигналов: Автореф. дис. канд.техн.наук: 05.12.01/ МЭИ. М., 1987. - 18 с.

4. Марченко Б.Г. Лінійні періодичні процеси // Пр. Ін.-ту електродинаміки НАН України. Електротехніка. - 1999. - С. 165-182. 


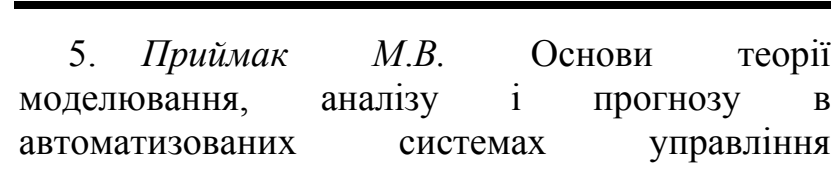
ритмічними процесами: Автореф. дис. докт.техн.наук:05.13.06/ Київ, НАУ,2001.-34 с.

6. Лупенко С.А., Приймак М.В., Щербак Л.M. Поліноміальний стохастично періодичний функціонал другого порядку як модель циклічних випадкових сигналів в нелінійних системах // Вимірювальна та обчислювальна техніка в технологічних процесах.Хмельницький: Навчальна книга. - 2000. - №1. C. 134-137.

7. Данилов Л.В., Матханов П.Н., Филиппов $E . C$. Теория нелинейных электрических цепей.Л.: Энергоатомиздат, 1990. - 256 с.

8. Мармарелис П., Мармарелис В. Анализ физиологических систем. Метод белого шума. М.: Мир, 1981. - 480 с.

9. Ку И.Х., Вольф А.А. Применение функционалов Вольтерра-Винера для анализа нелинейных систем // Техническая кибернетика за рубежом / Под. ред. В.В. Солодовникова - М.: Машиностроение, 1968. - С. 145-165.

10. Марченко Б.Г. Метод стохастических интегральных представлений и его приложения в радиотехнике. - К.: Наукова думка, 1973. - 192 с.

11. Красильников О.I., Марченко Б.Г., Приймак M.B. Процеси 3 незалежними періодичними приростами і періодичні білі шуми // Відбір і обробка інформації. - 1996. Вип. 10. C. 22-27.

12. Приймак М.В. Аналіз енергонавантажень iз використанням лінійних випадкових процесів // Вісник Тернопільського держ. техн. ун-ту. 1999.- том 4, число 1. - С.84-87.

\section{Приймак Микола Володимирович.}

Місце роботи: Тернопільський державний технічний університет, кафедра комп'ютерних наук.

Вчена ступінь: доктор технічних наук.

Вчене звання: доцент.

Посада: зав. кафедрою комп'ютерних наук Тернопільського державного технічного університету.

Наукові інтереси: періодичні білі шуми, марківські періодичні процеси, математична статистика в технічних застосуваннях;

Християнство в Україні, його вплив на духовний $i$ економічний стан держави, відповідальність духовенства перед Богом та народом за цей стан і шляхи виходу з нього.

\section{Лупенко Сергій Анатолійович}

Місце роботи: Тернопільський державний технічний університет, кафедра комп'ютерних наук.
Вчена ступінь: кандидат технічних наук.

Посада: доцент кафедри комп'ютерних наук Тернопільського державного технічного університету.

Наукові інтереси: лінійні випадкові процеси $i$ поля, моделювання нелінійних динамічних систем. 